\title{
Identifiability of Tyre Force Contact Prediction from Defor- mation Measurements
}

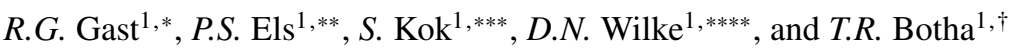 \\ ${ }^{1}$ Department of Mechanical and Aeronautical Engineering, University of Pretoria \\ Private Bag X20, Hatfield 0002, South Africa
}

\begin{abstract}
The possibility of accurately inferring the external forces applied to a vehicle can directly contribute to better safety systems and thus lowers the chance of injury or loss of life. These external forces are applied to a vehicle through the tyres and are challenging to measure directly. Still, it is possible to measure acceleration, deformation, or strain on the inner surface of a tyre. These measurements are theorized to be strongly linked to the forces produced by the tyre. However, it is still unknown whether or not one can always identify external forces from internal measurements in this way. Research has mainly focused on obtaining estimates of tyre forces rather than establishing to what extent these tyre forces are identifiable. This paper investigates this by conducting a virtual experiment that simulates known external forces applied to the tyre and computes the strains and displacements inside the tyre. A virtual inverse simulation then recovers the external forces from either the deformation or strain computed on the inside of the tyre. The identifiability of the forces recovered by the virtual inverse simulation is investigated by adding artificial measurement noise and initial guess perturbations to quantify the variance in the identified forces.
\end{abstract}

\section{Introduction}

Vehicle safety is improved with the addition of Advanced Driver Assistance Systems which take over from human inputs to prevent or reduce the effect of accidents. These systems rely on estimates of the vehicle state, and thus better control is correlated with better estimates. Tyre forces are the primary external forces applied to a vehicle, especially at lower speeds, but are notoriously difficult to determine. One possibility for determining tyre force estimates requires "intelligent" or "smart" tyres, which have embedded sensors that are used to infer the tyre force - from acceleration, strain or optical sensors [1]. Of all the sensing options, an optical approach is the most attractive as the full-field deformation and strain field of the inner surface of the tyre can be measured. Both these fields are theorized to be strongly correlated to the external forces applied to the tyre. Previous work at the University of Pretoria on an

\footnotetext{
*e-mail: robin.gast@ tuks.co.za

**e-mail: schalk.els@up.ac.za

***e-mail: schalk.kok@up.ac.za

****e-mail: nico.wilke@up.ac.za

†e-mail: theunis.botha@up.ac.za
} 
intelligent tyre concept has lead to the development of the T2-Cam (Tyre-Terrain Camera) system, which uses stabilized stereo-vision cameras to record the inner surface of a rolling tyre [2]. Digital Image Correlation is used to determine the 3D deformation field from a pair of images captured by T2-Cam. The surface strains can be calculated from a pair of reference images of the inside of the undeformed, inflated tyre [2, 3]. Most recently, Pegram et al. showed agreement between point measurements of the strains on the inner surface of the tyre from strain gauges and those measured by T2-Cam [4]. There is limited research regarding whether or not these systems can be used to identify tyre force in general rather than in specific cases. It is unknown whether a measured displacement or strain field corresponds to a unique force distribution at the tyre-road interface. This presents a challenge for developing a model to predict tyre forces from measurements. In this study, a virtual experiment simulates known external forces applied to the tyre and computes the strains and displacements inside the tyre. A virtual inverse simulation is then conducted to investigate the identifiability of the external forces given either the computed deformation or strain fields on the inside of the tyre. The study includes aleatoric uncertainty by adding artificial measurement noise and initial guess perturbations to quantify the variance in the identified forces.

There is little research regarding whether or not these systems can be used to identify tyre force in general rather than in specific cases, and it is not currently known whether a measured displacement or strain field corresponds to a unique force distribution at the tyreroad interface. This presents a challenge for developing a model to predict tyre forces from measurements. In this work a virtual inverse simulation is solved to recover forces on the outside of a tyre, which correspond to either a deformation or strain field virtually measured on the inner surface of the tyre.

In Section 2 the process of investigating the possibility of determining external tyre forces from internal measurements is described using a standard finite element code. A suitable candidate tyre is chosen to base the study on for which an appropriate axis system is defined. The process of acquiring geometry is described and a "test section" is defined to simplify the study. Section 2.4 describes the development of a coarse finite element model of the tyre which is used to generate virtual experiments for the inverse analysis that follows. An optimisation-based approach to identify tyre forces is described in Section 2.5 using the same previously-described model, and is then used to quantify the sensitivity of the problem to measurement noise and an initial guess in Section 3. The results are discussed in Section 4 and show that the identifiability of total forces and moments is high even in the presence of measurement noise and a poor initial guess, but the distribution of forces can not be identified if the initial guess is poor. Therefore in Section 5 it is recommended for future work to focus on regularization methods.

\section{Method}

\subsection{The tyre investigated}

To simplify the problem, a tyre is investigated which does not have steel bands running from bead-to-bead and, therefore, will have macroscopic material properties closer to rubber. Additionally, the effects of tread block deformations are removed by choosing a slick tyre with no tread blocks. The tyre being studied is a Trelleborg TM700 280/70R16 agricultural tyre (Figure 1), the same tyre used by Pegram et al. [4] except with the tread lugs shaven off. This tyre does not have steel cords running from bead-to-bead and is, therefore, suitable for the analysis. The tyre geometry has already been captured with a combination of profile gauges and laser scanning and converted into a Computer-Aided Design (CAD) model for previous studies 


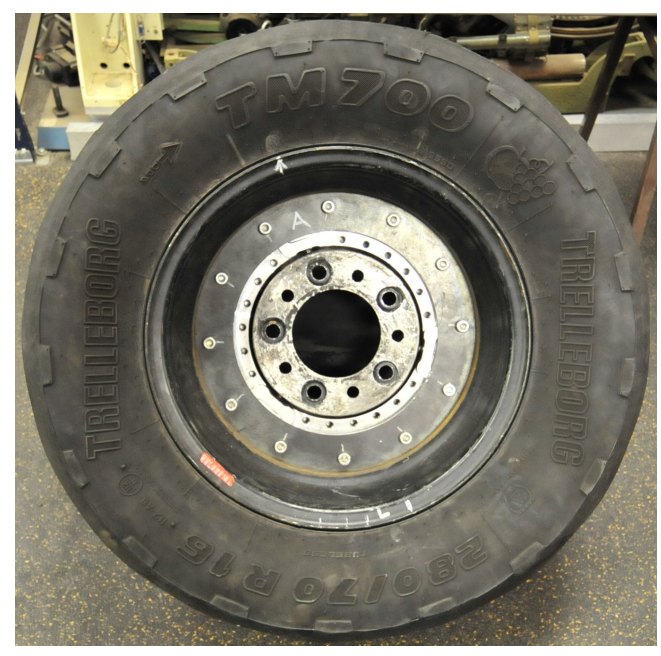

Figure 1: A Trelleborg TM700 with the tread lugs removed.

\subsection{Axis System}

The description of forces and moments transferred to or from a tyre requires defining an appropriate axis system. For the investigation of tyre behaviour, an axis system that is centred around the tyre is preferred. For this study, the TYDEX (TYre Data EXchange Format) CAxis system is adopted as shown in Figure 2. In the TYDEX C-axis system, the X-axis is defined as the line in the wheel plane parallel to the ground plane. The $y$-axis is perpendicular to the wheel plane and therefore is the spin axis, and the z-axis follows from the right-hand rule. The slip angle is the included angle between the tyre heading and the direction of travel. The tyre inclination, or camber angle, is also shown. This is the angle between the ground plane normal and the tyre spin axis normal.

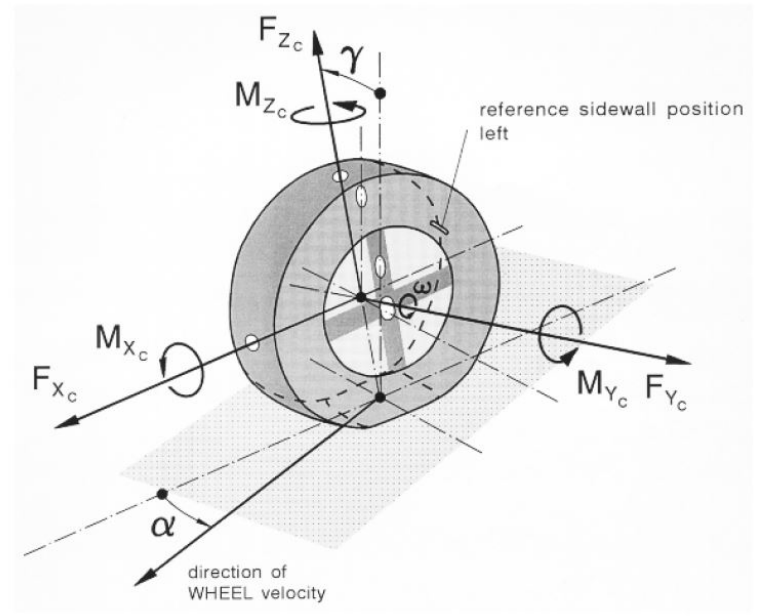

Figure 2: TYDEX C tyre axis system - adapted from Van Oosten et. al. [5] 


\subsection{Geometry}

The tyre inflation pressure places it in a state of tension and allows the tyre carcass to bear significant loads as it acts as a ring under tension. The portion of the sidewall which is opposite an applied radial load is in further tension, and contributes significantly to the vertical stiffness of an inflated tyre. The portion of the sidewall adjacent to radial loads can deform significantly as it naturally buckles, but it does not contribute substantially to the stiffness. This region near to the road will be studied without the inflation effects present, as it is theorized that this represents a scenario in which the applied forces are more readily identified than one in which the aforementioned effects of inflation and the sidewall stretching are present. This is done by modelling the problem as a $90^{\circ}$ arc of the tyre which is in contact with the road surface and subject to no internal pressure.

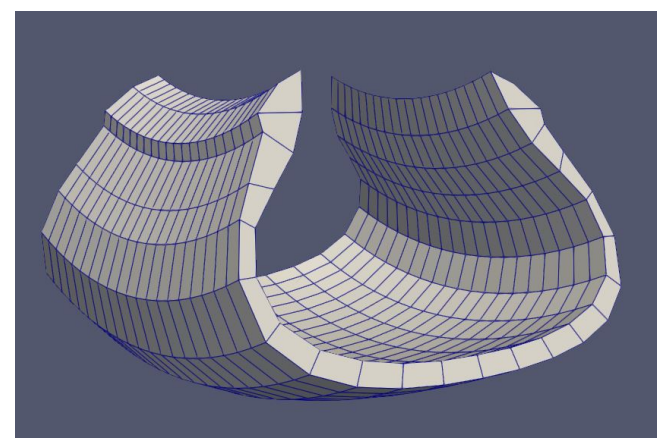

Figure 3: Geometry of the test section.

\subsection{Virtual Experiments}

One can use a virtual experiment to generate plausible inputs for the virtual inverse simulation. This virtual experiment must reflect the reality of measuring deformations and strains on the inner surface of a tyre, and therefore one must understand the experimental setup used to do this. Laboratory tests using T2-Cam allow for the measurement of strain $\left(\epsilon_{x x}, \epsilon_{y y}, \epsilon_{x y}\right)$ and deformation $\left(U_{x}, U_{y}, U_{z}\right)$ on the inside of the tyre using stereo-vision cameras The total forces and moments acting on the tyre for a particular loading condition can be measured with a wheel-force transducer. The distribution of the contact forces between the tyre and the contact surface are measured in the vertical direction in the laboratory with a Tekscan Pressure Mapping Sensor 8001 [6], but sensors for measuring the longitudinal and lateral pressures are not widely available. Estimating the distribution of these forces may significantly impact other research areas, such as terramechanics, where the ability to predict both the pressure and sinkage of a tyre substantially improves the possibility of predicting tractive effort. Since it is not practical to measure the distribution of the forces on a tyre, this study is instead conducted using "virtual experiments". A Finite Element model of the test section is used to generate plausible displacements and strains on the inside of the tyre given some loading condition. This is limited to the quasi-static loading of the un-inflated test section against a smooth, flat surface with hard contact. This loading condition generates loads in the z-direction only. The open-source FEM code CalculiX [7] was chosen to perform the virtual experiments. These virtual experiments will be used as a proof of concept to determine to what degree the forces produced by a tyre can be identified by the displacement or 
strains measured inside the tyre. The test case allows for one to investigate this with some simplifications.

\subsubsection{Pre-Processing}

The mesh of the test section is generated with the open-source tool Gmsh [8] with 440 incompatible mode eight-note brick elements (C3D8I) to facilitate using a non-linear hyperelastic material model. The material is modelled as Neo-Hookean with an equivalent Young's Modulus of $10 \mathrm{MPa}$ and a Poisson's ratio of $v=0.499$. The material was chosen to be on the low end of the scale of typical rubber stiffness's found in tyres, as it is expected that it will better expose the sensitivity of the problem. The boundary conditions at the bead are modelled as a fixed displacement, which has been shown to have a negligible effect on the tyre's vertical stiffness compared to a full contact simulation of the rim-bead interaction [9]. The boundary condition between the tyre and the road is iteratively applied by first displacing the node on the tread which most violates the road for a given total displacement, running this simulation to determine the new penetration with the road, and then repeating this process until no tread nodes penetrate the road. If a node presents an opposing reaction force in the $\mathrm{z}$-direction at any stage, it is released. This simulation allows one to determine the strains and displacements inside the tyre, given some loading, representing what one can measure in the laboratory on an actual tyre.

\subsubsection{Typical Virtual Experiment Results}

Figure 4 shows various results of a virtual experiment in which the test section was displaced $30 \mathrm{~mm}$, which corresponds to typical displacements that the tyre might sustain under heavy loads. Figures $4 \mathrm{a}$ and $4 \mathrm{c}$ indicate that in the absence of inflation pressure, the test section bulges in the middle, causing a loss of contact in the centre of the test section. The reaction forces at the tyre tread resulting from the displacement are shown in Figure 4b, where the total vertical reaction force was $359 \mathrm{~N}$ spread across two distinct areas of contact. The average nodal force over these nodes in contact is $25.68 \mathrm{~N}$ with a standard deviation of $7.27 \mathrm{~N}$. Figures $4 \mathrm{~d}, 4 \mathrm{e}$ and $4 \mathrm{f}$ show the three components of percentage surface strain calculated from the full-field displacement measurements. These indicate how rapidly the strain dissipates from the inside of the tyre to the outside of the tyre.

\subsection{Virtual Inverse Simulations}

Given some measured displacement $\boldsymbol{U}$ or strain $\boldsymbol{\epsilon}$ inside the tyre, the corresponding external forces, $\boldsymbol{x}$, that caused them must be recovered. The challenge is that the forces must be recovered at a different location to where the displacements and strains are measured. One can investigate the identifiability of these forces with a similar Finite Element model but instead prescribing forces at the tread instead of a displacement and attempt to reproduce the displacements and strains that were "measured" in the virtual experiment.

An optimization-based approach is suitable for iterating through applied forces on a FEM model until the measured displacements and strains match and is an appropriate tool for determining the sensitivity of the problem. An initial guess, $\boldsymbol{x}_{0}$, of the external forces applied to the outside of the tyre is required to solve the virtual inverse problem. The resulting displacements or strains are computed and compared to those computed in the virtual experiment. When good agreement is found the procedure terminates, else the optimizer updates the forces and repeats the process until convergence is reached. The locations at which forces 


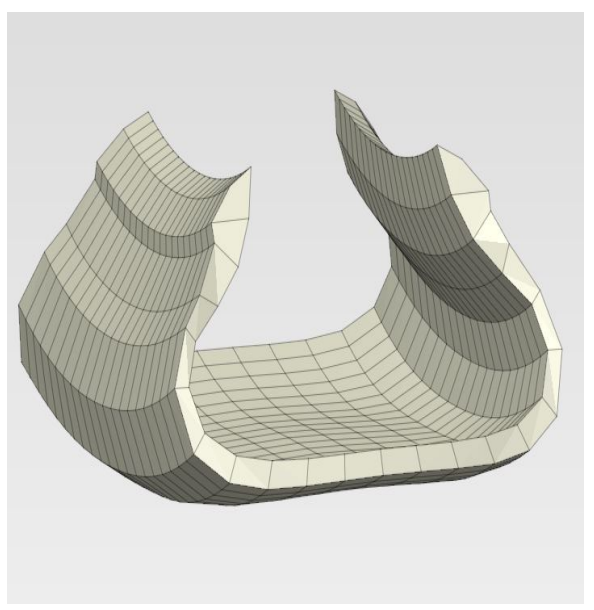

(a) Deformed geometry.

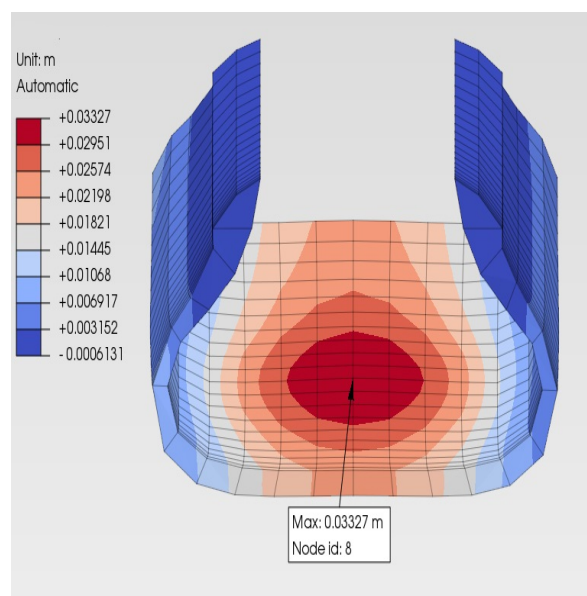

(c) Vertical deformation.

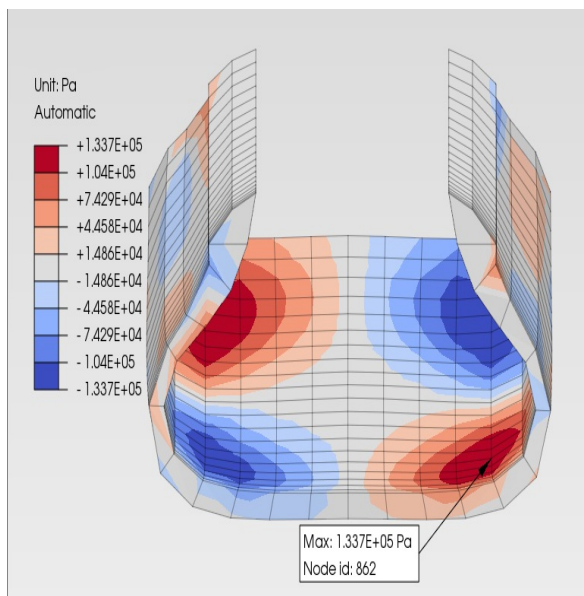

(e) $\epsilon_{x y}$.

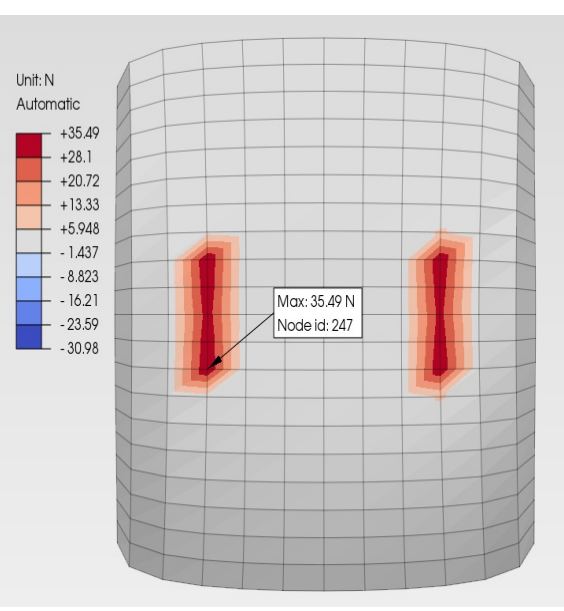

(b) Vertical reaction forces.

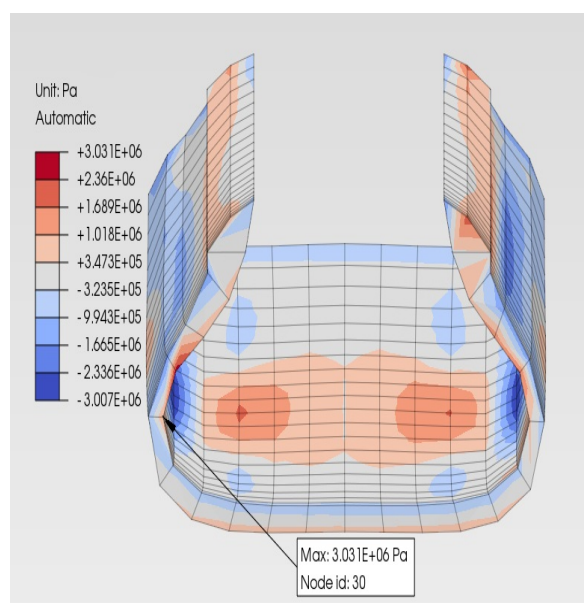

(d) $\epsilon_{x x}$.

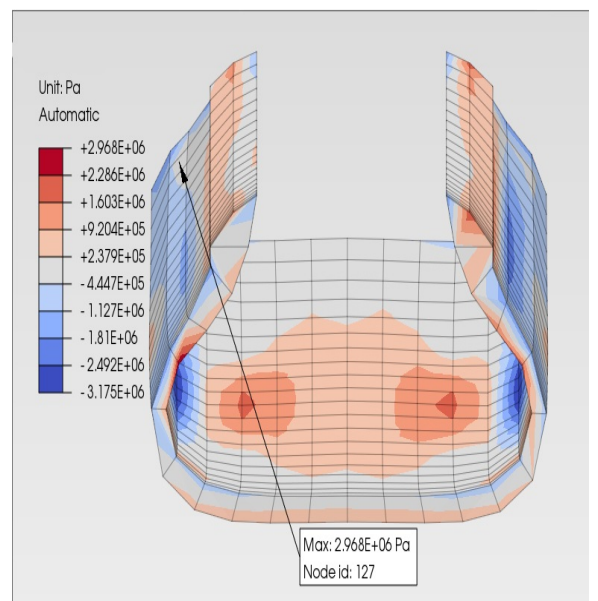

(f) $\epsilon_{y y}$.

Figure 4: Results from the virtual experiment for a 30mm displacement. 
can be applied are restricted to those where contact does occur to reduce the dimensionality of the problem from 210 unknowns to 14. The unconstrained Nelder-Mead optimization algorithm is used to solve the optimization problems

$$
\begin{gathered}
\underset{\boldsymbol{x}}{\operatorname{minimize}} f(\boldsymbol{x})=\sqrt{\frac{\sum\left(U_{x}-U_{x}^{*}(\boldsymbol{x})\right)^{2}+\sum\left(U_{y}-U_{y}^{*}(\boldsymbol{x})\right)^{2}+\sum\left(U_{z}-U_{z}^{*}(\boldsymbol{x})\right)^{2}}{N}}, \text { and } \\
\underset{\boldsymbol{x}}{\operatorname{minimize}} f(\boldsymbol{x})=\sqrt{\frac{\sum\left(\widetilde{\epsilon_{x x}}-\widetilde{\epsilon_{x x}^{*}}(\boldsymbol{x})\right)^{2}+\sum\left(\widetilde{\epsilon_{y y}}-\widetilde{\epsilon_{y y}^{*}}(\boldsymbol{x})\right)^{2}+\sum\left(\widetilde{\epsilon_{x y}}-\widetilde{\epsilon_{x y}}{ }^{*}(\boldsymbol{x})\right)^{2}}{N}}
\end{gathered}
$$

where $\boldsymbol{U}$ and $\boldsymbol{\epsilon}$ are the $N$ nodal displacements $\left(U_{x}, U_{y}, U_{z}\right)$ and $N$ nodal strains $\left(\widetilde{\epsilon_{x x}}, \widetilde{\epsilon_{y y}}, \widetilde{\epsilon_{x y}}\right)$ on the inner surface of the test section from the virtual experiment, while $\boldsymbol{U}^{*}(\boldsymbol{x})$ and $\boldsymbol{\epsilon}^{*}(\boldsymbol{x})$ are the $N$ nodal displacements and $N$ nodal strains $\left(\widetilde{\epsilon_{x x}}(\boldsymbol{x}), \widetilde{\epsilon_{y y}}(\boldsymbol{x}), \widetilde{\epsilon_{x y}}(\boldsymbol{x})\right)$ on the inner surface of the current iteration of the virtual inverse problem. The nodal strains $\left(\left(\widetilde{\epsilon_{x x}}, \widetilde{\epsilon_{y y}}, \widetilde{\epsilon_{x y}}\right),\left(\widetilde{\epsilon_{x x}}(\boldsymbol{x}), \widetilde{\epsilon_{y y}}(\boldsymbol{x}), \widetilde{\epsilon_{x y}}(\boldsymbol{x})\right)\right)$ are extrapolated from the strains at the integration points of each element to the nodes on the inner surface of the tyre because T2-Cam reports surface strains. The design vector $\boldsymbol{x}$ is the forces applied to the outside of the test section, and $f(\boldsymbol{x})$ is the objective function used in the optimization. Equations 1 and 2 are displacement-based and strain-based virtual inverse problems treated as different methods in this study but can be combined into one objective function for a third formulation.

The sum of design vector $\boldsymbol{x}$ which is solved for in the virtual inverse problem is the total force acting on the tyre, $F_{z}$, and each force in the design vector produces can produce up two moments $M_{x}, M_{y}$. If the total vertical force recovered in the virtual inverse simulation correlates with the appropriate virtual experiment, then it indicates that the forces that were recovered are statically equivalent to the true answer. The sum of each of these moments $M_{x}, M_{y}$ is close to zero in the virtual experiment therefore if the result of a virtual inverse simulation corresponds to this it indicates that on average the forces are symmetrical but may still vary. The design vector $\boldsymbol{x}$ is a distribution of force and has a particular shape, which is desirable to recover in addition to the total forces and moments. This distribution of forces recovered from the virtual inverse simulation can be compared to the virtual experiment as a distribution of errors. A distribution of errors which is zero-mean with a low standard deviation relative to the largest nodal force predicted by the virtual experiment indicates that the design vector $\boldsymbol{x}$ is a similar distribution to the true answer.

\section{Results}

\subsection{Ideal Virtual Inverse Simulations}

The best-case scenario to recover forces from measured displacements or strains would occur if there is no appreciable measurement noise and the initial guess is good. Such a scenario is a useful baseline for comparisons to inverse solutions that follow. Two virtual inverse simulations like this were run, one using the displacement-based method and the other with the strain-based method. In both cases the initial guess was chosen to be $90 \%$ of the known answer from the virtual experiment and therefore has the correct shape but lower magnitude so as to avoid numerical instability. Figure 5 shows the results of solving for the contact forces using both virtual inverse methods. The net force in the displacement-based method was $359 \mathrm{~N}$ and $357 \mathrm{~N}$ for the strain-based method. The standard deviation of the distribution of 
forces from the virtual experiment was $7.27 \mathrm{~N}(25.68 \mathrm{~N}$ mean $)$, while that of the displacementbased and strain-based methods was $7.39 \mathrm{~N}(25.66 \mathrm{~N}$ mean $)$ and $7.37 \mathrm{~N}(25.52 \mathrm{~N}$ mean $)$ respectively. In both cases, the objective function converged to a zero error (within the limits of machine precision), and although the distribution of the force is not perfect, it is a reasonable reflection of the expected forces. In both cases a small moment about $\mathrm{x}$ and $\mathrm{y}$ was present due to the errors in the force distribution, however, they are all under $0.9 \mathrm{Nm}$.

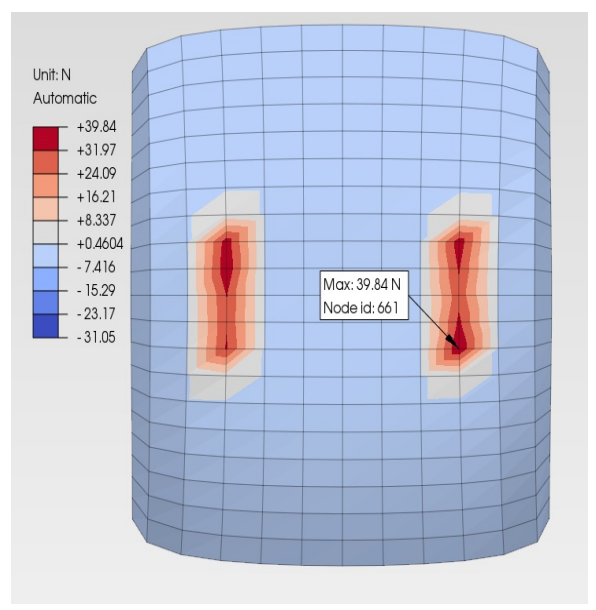

(a) Displacement-based.

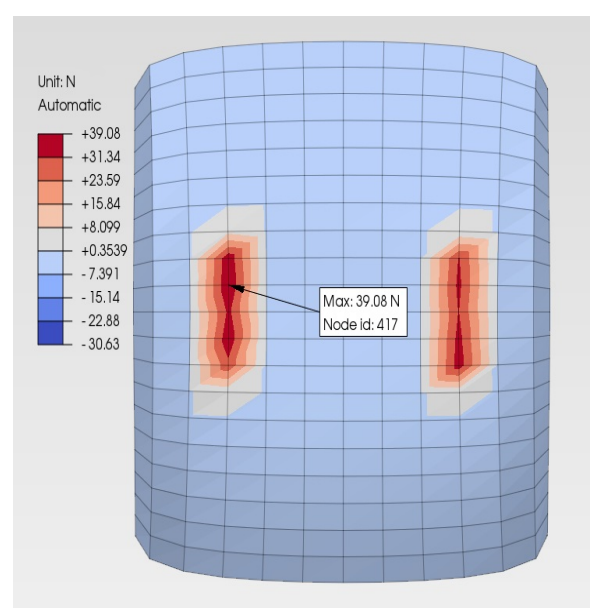

(b) Strain-based.

Figure 5: Identified Vertical Reaction Forces.

\subsection{Effects of Measurement Noise}

As this study is focused on the identifiability of the forces resulting from a virtual inverse simulation, comparisons between the two methods will be made for comparable levels of uncertainty. The effect of these uncertainty levels was investigated by sampling from zeromean $1 \mathrm{~mm}$ or $0.04 \%$ standard deviation for the displacement-based and strain-based virtual inverse simulations, respectively. This noise was added to the appropriate measurement from the virtual experiment. The current strain or displacement is compared to a noisy version of the virtual experiment at each iteration. The initial guess remained as $90 \%$ of the known answer from the virtual experiment, as the intent of this portion of the study is aimed at understanding the effect of noise on the identifiability of external forces.

Table 1 shows the total vertical force and both moments $\left(M_{x}, M_{y}\right)$ from the virtual experiment and the predictions from both virtual inverse simulations with and without measurement noise. The forces and moments for the noisy simulations were averaged over 10 runs. This level of noise does not significantly impact the forces' identifiability, since the total vertical force is predicted well, and there are negligible moments predicted where one expects zero moments. Table 2 shows the RMS error in the displacement and strain inside the test section with and without additive noise. In cases where the displacement-based formulation was used, the RMS error on the strains is not significantly worse than if a strain-based method is used and vice-versa.

The force error at each node from the virtual inverse simulation to the reference force from the virtual experiment is calculated for both the strain and deformation formulations. 
A histogram of these errors is presented in Figure 6. Both distributions are zero-mean, but the displacement-based method's standard deviation was $2 \mathrm{~N}$ whilst that of the strain-based method was $3.2 \mathrm{~N}$.

Table 1: Forces and moments predicted by virtual inverse simulations with and without noise.

\begin{tabular}{llll}
\hline & $F_{z}$ & $M_{x}$ & $M_{y}$ \\
& $\mathrm{~N}$ & $\mathrm{Nm}$ & $\mathrm{Nm}$ \\
\hline Reference & 359.08 & 0 & 0 \\
Displacement-based & 359.23 & -0.840 & -0.019 \\
Strain-based & 356.71 & -0.15 & -0.25 \\
Displacement-based $+\mathcal{N}(0,1)$ & $358.28 \pm 0.4$ & $-0.84 \pm 0.1$ & $-0.020 \pm 0.05$ \\
Strain-based $+\mathcal{N}(0,0.04)$ & $357.40 \pm 0.9$ & $-0.2 \pm 0.02$ & $-0.27 \pm 0.01$ \\
\hline
\end{tabular}

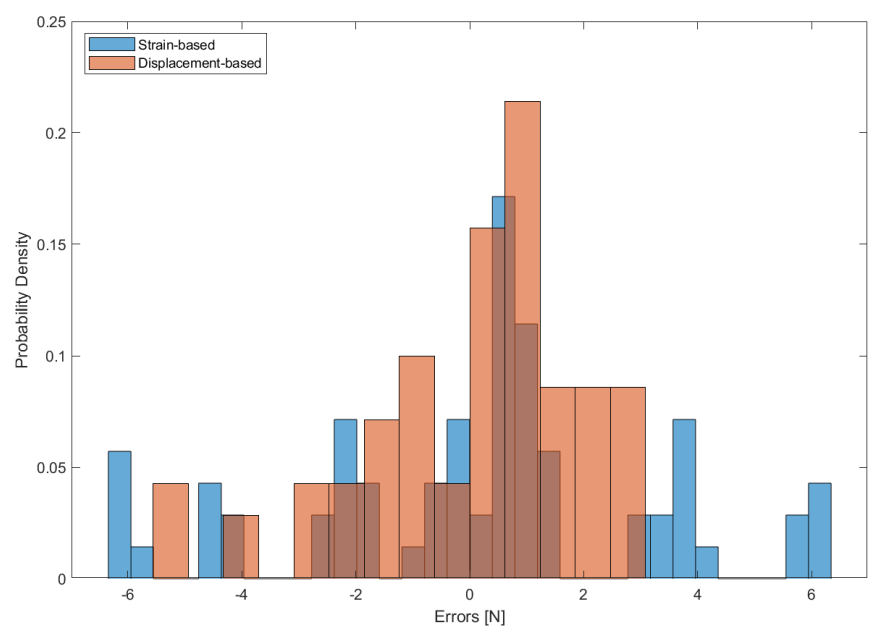

Figure 6: Spread of the normalized errors on the forces recovered by the virtual inverse simulations.

Table 2: Average root mean square error for the virtual inverse simulations with and without measurement noise.

\begin{tabular}{lllllll}
\hline & $\begin{array}{l}U_{x} \\
\mathrm{~mm}\end{array}$ & $\begin{array}{l}U_{y} \\
\mathrm{~mm}\end{array}$ & $\begin{array}{l}U_{z} \\
\mathrm{~mm}\end{array}$ & $\begin{array}{l}\epsilon_{x x} \\
\mu\end{array}$ & $\begin{array}{l}\epsilon_{y y} \\
\mu\end{array}$ & $\begin{array}{l}\epsilon_{x y} \\
\mu\end{array}$ \\
\hline Displacement-based & 0.0075 & 0.012 & 0.049 & 116.60 & 172.07 & 138.53 \\
Strain-based & 0.030 & 0.19 & 0.24 & 485.70 & 560.16 & 252.52 \\
Displacement-based $+\mathcal{N}(0,1)$ & 0.010 & 0.040 & 0.070 & 131.08 & 171.22 & 147.54 \\
Strain-based $+\mathcal{N}(0,0.04)$ & 0.27 & 1.93 & 2.25 & 4538.7 & 5089.14 & 2530.81 \\
\hline
\end{tabular}




\subsection{Effects of Initial Guess}

Suppose the design domain contains many local minima. In that case, an initial guess which is close to the answer will not correctly expose the sensitivity of the forces recovered by the virtual inverse problem. To investigate these, ten runs are performed for each method where the initial guess is randomized but remains on average $90 \%$ of the answer. For each virtual inverse simulation, samples drawn from a Gaussian random variable with zero-mean and $4 \mathrm{~N}$ standard deviation are added to the initial guess, after which the usual procedure is followed. The standard deviation of the uncertainty added to the initial guess is more than $10 \%$ of the maximum nodal force in the virtual experiments of $35.5 \mathrm{~N}$. In addition, similar virtual inverse simulations are run with a "uniform" initial guess, where the initial guess is the same at every node and corresponds to the test section's vertical stiffness multiplied with the total displacement and divided by the number of nodes in contact. The uniform case is statically equivalent to the true answer but can severely over- or under-estimate local forces at a particular node. The same level of artificial measurement noise used in Section 3.2 was retained for all simulations.

Table 3 shows the average vertical force and moments predicted by the virtual inverse simulations over ten runs, each with two different initial guesses. Although both guesses for both methods show larger moments than when an ideal initial guess is used, the total vertical force is still predicted within $6 \mathrm{~N}(1.6 \%)$ in all cases. Table 4 shows that both initial guesses for both methods converged to solutions that have very low RMS error on the displacement and strains on the inside of the test section, regardless of whether a displacement-based or a strain-based method was used. However, when considering the force distributions shown in the histograms of the normalized errors, the variance in the forces predicted locally has become very large. Figure 7 a shows that an initial guess of $90 \%$ of the true forces with a large uncertainty still produces a zero-mean distribution of normalized errors, but the variance is around four times as high. The standard deviation for the displacement-based and strainbased methods with this initial guess is $16.4 \mathrm{~N}$ and $11.6 \mathrm{~N}$, respectively. Figure $7 \mathrm{~b}$ shows that the variance is even worse when a uniform initial guess is used, and a bi-modal distribution with high variance appears. For the latter, he standard deviations of the normalized errors for the displacement-based and strain-based methods are $16.8 \mathrm{~N}$ and $13.3 \mathrm{~N}$, respectively.

Table 3: Forces and moments predicted by virtual inverse simulations with different initial guesses.

\begin{tabular}{lllll}
\hline & & $F_{z}$ & $M_{x}$ & $M_{y}$ \\
Initial Guess & Method & {$[\mathrm{N}]$} & {$[\mathrm{Nm}]$} & {$[\mathrm{Nm}]$} \\
\hline Reference & & 359.08 & 0 & 0 \\
$90 \%+\mathcal{N}(0,4)$ & Displacement & $358.6 \pm 4$ & $-0.933 \pm 1.3$ & $0.03853 \pm 0.4$ \\
$90 \%+\mathcal{N}(0,4)$ & Strain & $356.6 \pm 5$ & $-1.239 \pm 2$ & $-0.1884 \pm 1$ \\
Uniform $+\mathcal{N}(0,4)$ & Displacement & $364.0 \pm 2$ & $-1.694 \pm 2$ & $-0.6371 \pm 0.2$ \\
Uniform $+\mathcal{N}(0,4)$ & Strain & $357.1 \pm 2$ & $-1.659 \pm 0.5$ & $0.07057 \pm 0.4$ \\
\hline
\end{tabular}

\section{Discussion}

In Section 3.2, it is clear that measurement noise does not significantly impact the identifiability of the total vertical contact forces in this test case. For a noise level representative of the 
Table 4: Average root mean square error for the virtual inverse simulations with different initial guesses.

\begin{tabular}{llllllll}
\hline Initial Guess & Method & $\begin{array}{l}U_{x} \\
\mathrm{~mm}\end{array}$ & $\begin{array}{l}U_{y} \\
\mathrm{~mm}\end{array}$ & $\begin{array}{l}U_{z} \\
\mathrm{~mm}\end{array}$ & $\begin{array}{l}\epsilon_{x x} \\
\mu\end{array}$ & $\begin{array}{l}\epsilon_{y y} \\
\mu\end{array}$ & $\begin{array}{l}\epsilon_{x y} \\
\mu\end{array}$ \\
\hline $90 \%+\mathcal{N}(0,4)$ & Displacement & 0.03227 & 0.08697 & 0.1997 & 284.4 & 351.9 & 226.2 \\
$90 \%+\mathcal{N}(0,4)$ & Strain & 0.05158 & 0.4087 & 0.4987 & 911 & 1029 & 487.2 \\
Uniform $+\mathcal{N}(0,4)$ & Displacement & 0.1041 & 0.6276 & 0.8744 & 1569 & 1895 & 1082 \\
Uniform $+\mathcal{N}(0,4)$ & Strain & 0.0446 & 0.4657 & 0.4825 & 930 & 1027 & 401.8 \\
\hline
\end{tabular}

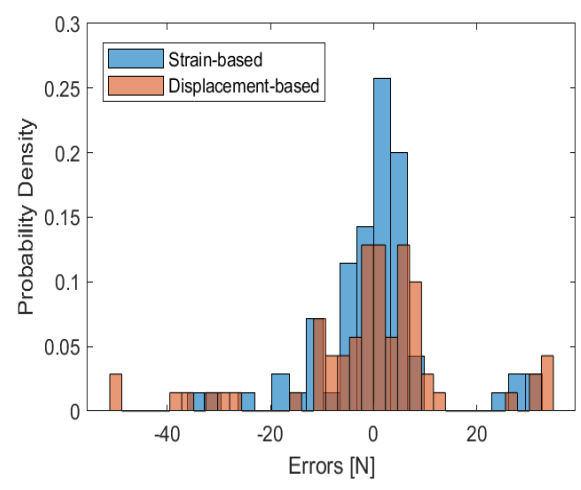

(a) $90 \%+\mathcal{N}(0,4)$.

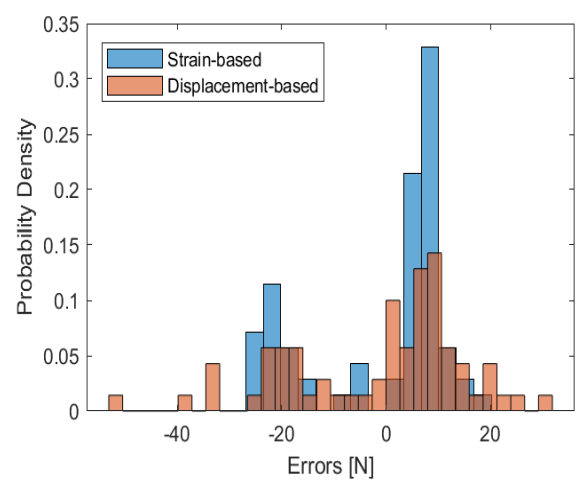

(b) Uniform guess $+\mathcal{N}(0,4)$.

Figure 7: Spread of the local normalized errors on the forces recovered by the virtual inverse simulations.

uncertainty in the deformation measurements, one can expect that the standard deviation of the uncertainty in the force distribution is less than $6 \%$ of the total force predicted for the test case. Comparable levels of uncertainty in the strain measurements produce slightly higher but similar levels of uncertainty in the force distribution at $9 \%$. In all cases, the total force predicted by the virtual inverse problem matches the virtual experiment. The standard deviation of the normalized local errors on the forces identified is within $10 \%$ of the maximum local force. For this case, it is possible to recover the force distribution over the contact patch.

Section 3.3 shows, however, that the identifiability of the individual contact forces diminishes significantly in the presence of a poor or non-physical initial guess. Despite this, the total force is still acceptably predicted by the virtual inverse problem, even when the distribution of those forces is far from the known solution from the virtual experiment. It is difficult to identify the true solution under non-ideal circumstances, especially if one does not have a good initial guess. Saint Venant's principle dictates that in this case, it becomes increasingly difficult to distinguish between different statically equivalent loads the further away the strains and displacements are observed [10].

The identifiability of the contact forces from deformation measurements is dominated more by the initial guess rather than the measurement noise. In Section 3.2, the initial guess is chosen with the correct shape but a lower magnitude than the true solution, while in Sec- 
tion 3.3, the initial guess can have a non-physical shape. However, the magnitude is still on average lower than the true solution. There is clearly some evidence that if one intends to recover the distribution of contact forces, then the initial guess must be regularized in some way to avoid converging to one of the many local minima that result in non-physical solutions in the solution space.

\section{Conclusions}

This work aimed to determine the degree to which external forces on a tyre can be identified from the deformations or strains on the inside of the tyre. In the ideal case, where no measurement noise is present, and the initial guess represents the true forces, both the distribution and the total forces on the tyre can be readily identified. If measurement noise is added, which corresponds to what can be expected in laboratory tests, then one can still expect to recover the total force on the tyre, but the distribution of these forces becomes harder to identify. Although the distribution of the forces is more difficult to identify when measurement noise is present, the spread of the errors on the forces is still low relative to the total force predicted. The identifiability of the distribution of the external forces becomes very low once the initial guess used in the virtual inverse problem is not representative of the true answer. However, it is still possible to accurately estimate the total force produced by the tyre in this case. These results indicate that there may be many local minima in the solution space of external forces, many of which have a similar total force but are non-physical for a tyre interacting with the road. It is recommended that future work focuses on methods for regularizing the initial guess used in such a way as to avoid converging to solutions where the distribution of forces is non-physical.

\section{References}

[1] The European Commission (2005). Intelligent tyre for accident-free traffic. Finland. https://cordis.europa.eu/project/id/IST-2001-34372.

[2] Gutherie, AG (2016) 3D Computer Vision Contact Patch Measurements Inside OffRoad Vehicle Tyres, Master's thesis, University of Pretoria.

[3] Feldesi, F (2018) Three-dimensional Contact Patch Strain Measurement Inside Rolling Off-Road Tyres, Master's thesis, University of Pretoria.

[4] Pegram, MS, Botha, TR and Els, PS (2021). Full-field and point strain measurement via the inner surface of a rolling large lug tyre. Journal of Terramechanics, 96, 11-22.

[5] van Oosten, JJM, Unrau, H-J, Riedel, A and Bakker, E (1997). TYDEX workshop: standardisation of data exchange in tyre testing and tyre modelling Vehicle System Dynamics, 1997, 272-288.

[6] Tekscan, Inc (2021). Accessed 1 July 2021, https://www.tekscan. com/products-solutions/pressure-mapping-sensors/8001.

[7] Dhondt, G and Wittig, K (1998) CalculiX: A Three-Dimensional Structural Finite Element Program.

[8] Geuzaine, C and Remacle, J-F (2009) Gmsh: A 3-D finite element mesh generator with built-in pre-and post-processing facilities International journal for numerical methods in engineering, 2009, 1309 - 1331.

[9] Stallmann, MJ (2013) Cost Effective FTire Parameterisation Methods for Ride Simulations with Large Off-The-Road Tyres, PhD Thesis, University of Pretoria.

[10] Gere, JM and Goodno, BJ (2013). Mechanics of Materials, 8th Eds. Cengage Learning. 